\begin{tabular}{|c|c|}
\hline Title & Interior thermal state of Enceladus inferred from the viscoelastic state of the ice shell \\
\hline Author(s) & Kamata, Shunichi; Nimmo, Francis \\
\hline Citation & $\begin{array}{l}\text { Icarus, 284, 387-393 } \\
\text { https://doi.org/10.1016ر.icarus.2016.11.034 }\end{array}$ \\
\hline Issue Date & 2017-03-01 \\
\hline Doc URL & http:/hdl .handle.net/2115/72823 \\
\hline Rights & $\begin{array}{l}\text { (C) } 2016 \text { Elsevier Inc. A ll rights reserved. Licensed under the Creative Commons A ttribution-NonCommercial- } \\
\text { NoDerivatives } 4.0 \text { International http://reativecommons.org/icenses/by-nc-nd/4.0/ }\end{array}$ \\
\hline Rights(URL) & http://creativecommons.org/icenses/by-nc-nd/4.0/ \\
\hline Type & article (author version) \\
\hline File Information & Kamata_and_Nimmo_2017_Icarus_final_draft.pdf \\
\hline
\end{tabular}

Instructions for use 


\title{
Interior thermal state of Enceladus inferred from the viscoelastic state of the ice shell
}

\author{
Shunichi Kamata ${ }^{\mathrm{a}, *}$, Francis Nimmo ${ }^{\mathrm{b}}$ \\ ${ }^{a}$ Creative Research Institution, Hokkaido University, Sapporo, Hokkaido 001-0021, Japan. \\ ${ }^{b}$ Department of Earth and Planetary Sciences, University of California, Santa Cruz, Santa \\ Cruz, CA 95064, USA.
}

\begin{abstract}
Recent geodetic measurements for Enceladus suggest a global subsurface ocean that is thicker beneath the south pole. In order to maintain such an ocean, viscous relaxation of topography at the base of the ice shell and melting of ice need to be balanced. In this study, we investigate the interior thermal state that can lead to the relaxation timescale being comparable to the melting timescale. Our results indicate that a basal heat flux about ten times higher than that due to radiogenic heating, or an ice shell tidal heating rate about ten times higher than the conventional estimate of $1.1 \mathrm{GW}$ is necessary if the ice shell is in thermal equilibrium. These requirements are concordant with recent astrometric studies.
\end{abstract}

Keywords: Enceladus; Planetary dynamics, Interiors

\section{Introduction}

Multiple flybys of the Cassini spacecraft revealed that the South Polar region of the Saturnian satellite Enceladus is one of the most geologically active regions among icy worlds (e.g., Spencer \& Nimmo, 2013). One striking result is that

5 Enceladus is currently producing plumes of gases and solid particles, strongly suggesting the presence of a subsurface ocean in Enceladus (e.g., Porco et al. 2006: Postberg et al., 2009, Waite et al., 2009). This result is surprising because

\footnotetext{
* Corresponding author

Email address: kamata@mail.sci.hokudai.ac.jp (Shunichi Kamata)
} 
such a small satellite (i.e., $\sim 250 \mathrm{~km}$ in radius) is expected to undergo ocean freezing on a timescale of order $10^{7}-10^{8}$ yr (Roberts \& Nimmo, 2008). Data from the infrared spectrometer onboard Cassini also revealed a hot south pole; the power output across this region is $\sim 5-15$ GW (e.g., Spencer et al. 2006; Howett et al., 2011). This amount of heat is much larger than the equilibrium value conventionally estimated considering tidal heating and radiogenic heating in the core (Meyer \& Wisdom, 2007, Roberts \& Nimmo, 2008). Despite a number of

15 studies since the discovery of plumes emanating from this region (e.g., Roberts \& Nimmo, 2008, Tyler, 2008; O'Neill \& Nimmo, 2010; Shoji et al., 2014, Travis \& Schubert, 2015, Roberts, 2015), the heat production mechanism that can account for this anomalously large amount of heat is still unclear. For a better understanding of Enceladus' heat budget, we investigate the current interior thermal state assuming an equilibrium condition as a starting point.

The thermal state inside an ice shell can be inferred from its viscoelastic state (e.g., White et al., 2013, Kamata \& Nimmo, 2014); warmer ice has a lower viscosity, leading to more rapid relaxation, and vice versa. Geodetic data of Enceladus obtained by the Cassini spacecraft suggest that the ice shell has a mean thickness of $\sim 25 \mathrm{~km}$ and is thinner around the South Polar Terrain (SPT) (Iess et al., 2014, McKinnon, 2015, Thomas et al., 2016, Van Hoolst et al., 2016. Čadek et al. 2016, Beuthe et al. 2016); the ice shell thus has large-scale topography not only at the surface (Nimmo et al., 2011) but also at its base. Since the basal temperature of the ice shell is the melting point, the basal viscosity of the shell would be low, and thus the timescale of viscous relaxation should be short. Lateral flow in the deep part of the ice shell should therefore efficiently remove basal topography, but this is apparently not consistent with the geodetic observations. A likely mechanism to maintain basal topography is melting of the shell near the SPT; the timescale of melting and that of topography relaxation 35 would need to be comparable.

Collins \& Goodman (2007) compare these two timescales assuming a regional subsurface ocean and find that such an ocean can be maintained. This result, however, is based on calculations assuming extremely large heat fluxes from the 
core; several GW of thermal emission are assumed at the base of the regional ocean. Although this assumption is consistent with observations at the surface, it is about ten times larger than radiogenic heat from the core. If the heat from the core is only due to radiogenic sources, is it possible to maintain a regionally thick subsurface ocean? If not, how large an amount of heat is necessary to maintain such an ocean? These questions remain uncertain.

In this study, we compare timescales for melting and relaxation under a wide variety of thermal conditions at the base of the ice shell. We assume that the ice shell is in a thermal equilibrium and is overlying a global subsurface pcean, which is suggested from a recent study of Enceladus' librations (Thomas et al. 2016). Section 2 describes our interior structure model for Enceladus.

50 Section 3 and 4 describe methods and results for the timescale for melting and that for relaxation, respectively. Section 5 compares these timescales and discusses implications for Enceladus' heat budget.

\section{Interior model}

We adopt a three-layer incompressible Enceladus model consisting of an ice 55 shell, a global subsurface ocean, and a rocky core. The layers are assumed to be a Maxwellian viscoelastic material, an inviscid fluid, and a purely elastic material, respectively. Table 1 lists the model parameters adopted. The background thickness of the ice shell $\left(D_{\mathrm{sh}}\right)$ is a free parameter and is less than $60 \mathrm{~km}$; otherwise the ice shell contacts the core. An increase in $D_{\text {sh }}$ requires an increase in the radius of the rocky core in order to lead to the same surface gravitational acceleration for a given satellite radius and given densities for each layer. Although these changes slightly affect the moment of inertia, our model gives a normalized moment of inertia of $\sim 0.33$, which is consistent with geodetic observations (Iess et al., 2014; McKinnon, 2015).

${ }_{65}$ We assume that the ice shell is completely (Airy) isostatically compensated. Topography at the base of the shell $\left(h_{b}\right)$ is then given by

$$
h_{b}=\frac{\rho_{i}}{\rho_{i}-\rho_{o}} \frac{g_{s} R_{s}^{2}}{g_{b}\left(R_{s}-D_{\mathrm{sh}}\right)^{2}} h_{s},
$$


where $h_{s}$ is surface topography, $\rho_{o}$ is density of ocean, $g_{s}$ is gravitational acceleration at the surface, $g_{b}$ is that at the base of the shell, and $R_{s}$ is the radius of Enceladus, respectively. The second factor represents the correction factor for the finite curvature. Note that our model considers the balance of vertical stresses only; this is different from a new isostatic model that minimizes deviatoric stresses (Beuthe et al. 2016). This difference, however, does not change the crustal structure significantly. We use the zonal components of surface topography given by Nimmo et al. (2011).

Figure 1 shows results for $D_{\mathrm{sh}}=20 \mathrm{~km}$. The location where the base of the shell becomes the deepest gives the extent of the thickened portion of the subsurface ocean. The volume of this portion $(V)$ ranges between $\sim 4.2 \times 10^{5} \mathrm{~km}^{3}$ (for $D_{\mathrm{sh}} \sim 60 \mathrm{~km}$ ) and $\sim 4.8 \times 10^{5} \mathrm{~km}^{3}$ (for $D_{\mathrm{sh}} \sim 10 \mathrm{~km}$ ). We note that $D_{\text {sh }}>9.5 \mathrm{~km}$ is necessary in order for the minimum shell thickness to exceed zero and $D_{\mathrm{sh}}<55.6 \mathrm{~km}$ to avoid contact between the shell and the silicate core. Consequently, the range of $D_{\text {sh }}$ we explore is between $9.5 \mathrm{~km}$ and $55.6 \mathrm{~km}$.

The viscosity profile of the ice shell is calculated from its temperature profile. In this study, we assume that the ice shell is in thermal equilibrium: heat from the core and heat production due to tides in the shell are balanced with heat transport due to thermal conduction. The temperature profile is given by

$$
0=\frac{1}{r^{2}} \frac{d}{d r}\left(k r^{2} \frac{d T}{d r}\right)+H(r),
$$

where $T$ is temperature, $k$ is thermal conductivity (taken to be constant), $r$ is radial distance from the center of Enceladus, and $H$ is heat production rate, respectively. The heat production rate $H$ for a Maxwell body is given by

$$
H(r)=\frac{2 \mu \bar{\varepsilon}^{2}}{\omega}\left(\frac{\omega \eta(r) / \mu}{1+(\omega \eta(r) / \mu)^{2}}\right),
$$

where $\mu$ is rigidity, $\eta$ is viscosity, $\omega$ is orbital frequency, and $\dot{\varepsilon}$ is strain rate, 90 respectively (Ojakangas \& Stevenson, 1989). See Table 1 for values we used. Temperature at the base of the shell $\left(T_{b}\right)$ and strain rate $(\dot{\varepsilon})$ are free parameters, and we adopt $180-270 \mathrm{~K}$ and $2 \times 10^{-10}-5 \times 10^{-10} \mathrm{~s}^{-1}$, respectively. Such values of strain rate give temperature profiles similar to those inferred from a numerical 
study coupling tidal heating and thermal convection at the SPT (Běhounková 95 et al. 2013).

We apply the rheology of pure water ice (Goldsby \& Kohlstedt, 2001) to this thermal profile and calculate the Newtonian viscosity as a function of depth:

$$
\eta(r)=\eta_{\mathrm{ref}} \exp \left(\frac{E_{a}}{R_{g}}\left(\frac{1}{T(r)}-\frac{1}{T_{\mathrm{ref}}}\right)\right)
$$

where $\eta_{\text {ref }}$ is reference viscosity of ice, $T_{\text {ref }}$ is reference temperature, $E_{a}$ is activation energy, and $R_{g}$ is the gas constant, respectively. Because the reference viscosity for the ice shell of Enceladus is uncertain, we consider a wide range of reference viscosities: $10^{12}-10^{17} \mathrm{~Pa}$ s.

Although the interior structure adopted in this study is simple, particularly below the ice shell, the timescale of viscoelastic relaxation at the base of the shell depends only weakly on the deep interior structure. Thus, the use of a more realistic deep interior structure model would not change our conclusions.

It is noted that we assume a purely conductive ice shell. The actual ice shell of Enceladus, however, may be convective, at least locally (e.g., Běhounková et al. 2013, 2015). If this is the case, the thickness of warm ice would be larger than in the conductive model. This would lead to a smaller relaxation timescale.

The consequence of this effect will be discussed in Section 5 .

\section{3. $\tau_{\text {melt }}$ : Timescale of melting}

The timescale of melting $\left(\tau_{\text {melt }}\right)$ can be estimated analytically:

$$
\tau_{\text {melt }}=\frac{\rho_{i} L V}{P_{\text {melt }}}
$$

where $\rho_{i}$ is density of ice, $L$ is latent heat of ice, $V$ is the volume of ice to be melted, and $P_{\text {melt }}$ is power used for melting, respectively. Results are shown in Figure 2 assuming that $V$ is the volume of the thickened portion of the ocean obtained in Section 2. The upper and lower bound is given by the largest and smallest $V$ (i.e., the smallest and largest $D_{\mathrm{sh}}$ ), respectively; the use of different values of $D_{\mathrm{sh}}$ has little effect on $\tau_{\text {melt }}$. For example, if $P_{\text {melt }}=1.1 \mathrm{GW}$, which is 
the conventional long-term limit of tidal heating rate (Meyer \& Wisdom, 2007), $\tau_{\text {melt }}$ is $\sim 4$ Myr.

\section{4. $\tau_{\text {rel }}$ : Timescale of viscoelastic relaxation}

\subsection{Method}

In contrast to the timescale of melting, that of viscoelastic relaxation $\left(\tau_{\text {rel }}\right)$ needs to be estimated numerically. We calculate the time evolution of topography at the base of the ice shell using a semi-analytical code (e.g., Kamata \& Nimmo, 2014). In the following, we briefly summarize the calculation method. The governing equation system consists of three equations: the linearized equation of momentum conservation,

$$
0=\nabla_{j} \cdot\left(\sigma_{j i}-P \delta_{j i}\right)+\rho \nabla_{i} \phi
$$

the Poisson's equation for the gravitational field,

$$
\nabla^{2} \phi=-4 \pi G \rho
$$

and the constitutive equation for a Maxwell medium,

$$
\frac{d \sigma_{j i}}{d t}+\frac{\mu}{\eta}\left(\sigma_{j i}-\frac{\sigma_{k k}}{3} \delta_{j i}\right)=\left(\kappa-\frac{2 \mu}{3}\right) \frac{d e_{k k}}{d t} \delta_{j i}+2 \mu \frac{d e_{j i}}{d t},
$$

where $\nabla_{i}$ is a spatial differentiation in direction of $i(=x, y, z), t$ is time, $\sigma$ is stress tensor, $e$ is strain tensor, $\phi$ is gravitational potential, $P$ is hydrostatic pressure, $\kappa$ is bulk modulus, $\mu$ is shear modulus, $\delta$ is the Kronecker delta, and $G$ is the gravitational constant, respectively. Applications of a finite difference to the time differentials in the constitutive equation and of a spherical harmonic expansion to the three equations leads to a six-component, time-dependent, inhomogeneous first-order ordinary differential equation system. We then carry out time-marching calculations and obtain the time evolution of the amplitude of topographic undulation for each harmonic degree. The time evolution of topography can be calculated by superposing spherical harmonics with timedependent coefficients. See Kamata et al. (2012) for further details of the formulation. In this study, we consider spherical harmonic degrees $n=1-10$. 
It should be noted that we assume a spherically symmetric interior model (see Section 2 and use a one-dimensional calculation code. Nevertheless, the Specifically, a factor of 10 increase in $\tau_{\text {rel }}$ can be achieved by a decrease in $T_{b}$ of 
10-20 K, depending on other parameters. In addition, a smaller $D_{\text {sh }}$ also leads to a longer $\tau_{\text {rel }} ; \tau_{\text {rel }}$ is almost proportional to $D_{\mathrm{sh}}^{-3}$, and this result is consistent with previous studies (e.g., McKenzie et al. 2000 Kamata et al., 2015b).

It is found that a change in $\dot{\varepsilon}$ has only a minor contribution. In our model, an increase in $\dot{\varepsilon}$ increases the amount of heat produced in the shell (see equation (3)). However, this heat becomes important only when the shell is soft; a low $\eta_{\text {ref }}$, a high $T_{b}$, and a large $D_{\text {sh }}$ are needed. Under such a condition, an increase in $\dot{\varepsilon}$ results in a shorter $\tau_{\text {rel }}$, though the effect is not significant.

It is noted that a low reference viscosity (i.e., $\eta_{\text {ref }} \sim 10^{13} \mathrm{~Pa} \mathrm{~s}$ ) requires a thin shell (i.e., $D_{\mathrm{sh}}<\sim 20 \mathrm{~km}$ ). This is because such a reference viscosity leads to a large tidal heating rate, and temperature significantly increases with depth. However, since the basal temperature is assumed to be the melting point, a thick ice shell is not allowed. The largest heat production rate is given when $\omega \eta / \mu=1$ (see equation 3); for our model, $\eta \sim 6 \times 10^{13}$ Pa s gives the largest heat production rate.

\section{Comparison of timescales and discussion}

Figure 5 summarizes the relation of the timescale of melting $\left(\tau_{\text {melt }}\right)$ and that of relaxation $\left(\tau_{\text {rel }}\right)$ under different thermal states at the base of the ice shell for a given power $\left(P_{\text {tot }}\right)$. Figure 5 (a) assumes that $P_{\text {tot }}$ is the sum of radiogenic heat $(0.3 \mathrm{GW})$ and tidal heat $(1.1 \mathrm{GW})$, both conventional estimates (Roberts \& Nimmo, 2008; Meyer \& Wisdom, 2007). Note that not all the power can be used to melt the ice; a part of heat is used to warm the shell (i.e., $H$ in equation (2)). See the Appendix for the method to calculate the amount of heat used for warming the shell. We subtract this power from $P_{\text {tot }}$ and calculate $\tau_{\text {melt }}$ using equation (5). We found parameter conditions that can lead to $\tau_{\text {rel }}=\tau_{\text {melt }}$ only if the basal heat flux $\left(q_{b}\right)$ is higher than $5 \mathrm{~mW} \mathrm{~m}^{-2}$ (the green shaded area in Figure 5); below this basal heat flux, relaxation is faster than melting, leading to rapid removal of basal topography. The radiogenic heat from the core of $0.3 \mathrm{GW}$ produces a heat flux of only $0.4-0.6 \mathrm{~mW} \mathrm{~m}^{-2}$ at the base of 
the ice shell, depending on $D_{\mathrm{sh}}$. Consequently, if the basal heat is only due to radiogenic sources, the basal topography would relax, and a regionally thick subsurface ocean cannot be maintained over the long term.

As noted above, our model assumes a purely conductive model, and a convective shell would lead to a shorter relaxation timescale. This indicates that the red shaded area in Figure 5 increases if we assume a convective ice shell; a minimum basal heat flux larger than our model would be necessary to maintain basal topography. Consequently, the occurrence of convection in the shell does not affect the above conclusion that a regionally thick subsurface ocean requires a heat source other than radiogenic. Furthermore, recent studies suggest that the present-day ice shell is thin (i.e., $\sim 20 \mathrm{~km}$ ) (e.g., Thomas et al., 2016 Van Hoolst et al. 2016, Čadek et al. 2016, Beuthe et al., 2016). Although mobile lid convection may be possible even for such a thin shell if the near-surface ice is sufficiently damaged (Barr, 2008), thermal convection for the entire ice shell is unlikely to operate; the ice shell is likely to be conductive.

Although we adopt an activation energy for pure water ice, an inclusion of other compounds may affect the "effective" activation energy for the actual ice on Enceladus. Hydrate salts have higher activation energies (Durham et al. 220 2005), leading to a larger increase in viscosity as decreasing temperature. However, such an effect becomes apparent only if the volume fraction of salts is $\geq 20 \%$ (Durham et al., 2005), which is unlikely to be the case for Enceladus. Consequently, our model (i.e., a pure water ice model) should give a good estimate of the relaxation timescale for the Enceladus' ice shell.

The inclusion of volatiles, such as ammonia, in ice also affects the physical properties of ice and ocean. When ammonia water freezes, ammonia molecules concentrate in the liquid. Consequently, melting and freezing leads to ammoniapoor ice and an ammonia-rich ocean. An increase in ammonia content in the ocean leads to a decrease in the melting point (i.e., the basal temperature). 230 Note that our calculation conditions cover basal temperatures down to $180 \mathrm{~K}$, which is close to the lowest melting point (e.g., Fortes \& Choukroun, 2010). Consequently, this effect does not affect our main conclusion that a regionally 
thick subsurface ocean requires a heat source other than radiogenic.

Under what conditions can basal topography be maintained? One possibility $10 \mathrm{GW}$. Thus, if the tidal heating rate in the shell is $\geq 10 \mathrm{GW}$, a regionally thick ocean can be maintained without invoking anomalously large heat production 
in the core. Shoji et al. (2014) show that episodically increased tidal heating though the amount of additional heat is only $\sim 0.5 \mathrm{GW}$. In addition, their study finds that an episodic heating cycle requires a basal temperature of $\sim 175 \mathrm{~K}$. Such a low melting point requires a large amount of ammonia (i.e., >30 wt\%) (e.g., Fortes \& Choukroun, 2010). However, this is difficult to reconcile with 2012). Roberts (2015) also points out that an increase in the amount of ammonia leads to a decrease in the density of water and that the $\mathrm{NH}_{3}$ content should be less than $\sim 15 \%$; otherwise ice I is not buoyant. Consequently, increased tidal heating due to coupled thermal-orbital evolution is unlikely to be the major librium estimate by Meyer \& Wisdom (2007) is based on the assumption that the dissipation factor $Q$ of Saturn is $>18,000$. This lower bound on $Q$ (assumed constant) is based on the present-day position and assumed primordial age of Mimas (e.g., Gavrilov \& Zharkov, 1977; Dermott et al., 1988). A smaller $Q$ cent interpretation of astrometric data suggests a $Q$ for Saturn about one order of magnitude smaller than the conventional estimate, which leads to a tidal heating rate of $\sim 15 \mathrm{GW}$ (Lainey et al., 2012). If this is the case, the maintenance of a regionally thick subsurface ocean can be explained. The problem is, however, a change in the $Q$ of Saturn affects not only the orbital evolution of Enceladus but also those of other satellites. In particular, such a low $Q$ is usually taken to require an extremely young age for inner satellites such as Mimas. However, this argument assumes that $Q$ is constant. Recent work has suggested both that the $Q$ of Saturn is highly frequency-dependent, and that the timescale of satel- 


\section{Conclusion}

The presence of a regionally thick, global subsurface ocean in Enceladus is inferred based on geodetic measurements by Cassini. This requires a balance between melting of ice and viscous relaxation of basal topography. We investigate the interior thermal state of Enceladus by comparing the timescale of melting of ice and that of viscoelastic relaxation at the base of the ice shell. Our results indicate that a heat flux $>5 \mathrm{~mW} \mathrm{~m}^{-2}(3 \mathrm{GW})$ at the base of the ice 
shell is necessary in order to maintain basal topography if we assume conventional estimates for radiogenic and tidal heating rates. This condition, however, is unlikely to be satisfied by Enceladus. Alternatively, we find that a tidal heating rate of $\sim 10 \mathrm{GW}$ in the ice shell is sufficient to maintain basal topography if the basal heat flux is due only to radiogenic heat from the core. Although we cannot rule out the possibility of episodic heat release, our results assuming thermal equilibrium are concordant with a large tidal heating rate supported by recent astrometric studies.

\section{Appendix: $P_{\mathrm{sh}}$ : Power used to heat the ice shell}

For a given shell thickness $\left(D_{\mathrm{sh}}\right)$, reference viscosity $\left(\eta_{\mathrm{ref}}\right)$, basal temperature $\left(T_{b}\right)$, and strain rate $(\dot{\varepsilon})$, we can calculate the temperature profile using equations (2) and (3). The power used to heat the ice shell $\left(P_{\mathrm{sh}}\right)$ is given as the integral of the tidal heating rate in the shell $(H)$ and can be expressed as

$$
P_{\mathrm{sh}}=\Omega\left(q_{s} R_{s}^{2}-q_{b} R_{b}^{2}\right)
$$

where $\Omega$ is solid angle of the heated region, $q_{s}$ is heat flux at the surface, $q_{b}$ is that at the base of the shell, $R_{s}$ is the radius of Enceladus, and $R_{b}=R_{s}-D_{\text {sh }}$ is the radial distance of the base of the shell from the center of Enceladus, respectively. Values except $\Omega$ can be determined from the temperature profile. The solid angle of the tidally heated region $(\Omega)$ is unclear. In this study, we use three different values for $\Omega$ : from the south pole to $75^{\circ} \mathrm{S}$, to $60^{\circ} \mathrm{S}$, and to $45^{\circ} \mathrm{S}$. Note that $P_{\mathrm{sh}}$ is large only if $H$ can be large. Such a case is found only for $\eta_{\text {ref }} \sim 10^{13} \mathrm{~Pa} \mathrm{~s}$ and $D_{\mathrm{sh}} \sim 20 \mathrm{~km}$ (see Figure 6). Most other conditions lead 345 to small $P_{\mathrm{sh}}$ independent of $\Omega$. Consequently, the use of different values for $\Omega$ does not change our results significantly.

\section{Acknowledgments}

We thank two anonymous reviewers for constructive comments improving the manuscript. Any requests for data produced in this study should be sent to 
Shunichi Kamata at kamata@mail.sci.hokudai.ac.jp. This work was supported by JSPS KAKENHI grant 16K17787.

\section{References}

Barr, A. C. (2008). Mobile lid convection beneath Enceladus'south polar terrain. J. Geophys. Res. Planets, 113. doi:10.1029/2008JE003114.

Běhounková, M., Tobie, G., Choblet, G., \& Čadek, O. (2013). Impact of tidal heating on the onset of convection in Enceladus's ice shell. Icarus, 226, 898904. doi:10.1016/j.icarus.2013.06.033

Běhounková, M., Tobie, G., Choblet, G., Čadek, O., Porco, C., \& Nimmo, F. (2015). Timing of water plume eruptions on Enceladus explained by interior viscosity structure. Nature Geosci., 8, 601-604. doi:10.1038/ngeo2475.

Beuthe, M., Rivoldini, A., \& Trinh, A. (2016). Enceladus's and Dione's floating ice shells supported by minimum stress isostasy. Geophys. Res. Lett., (pp. n/a-n/a). doi:10.1002/2016GL070650.

Čadek, O., Tobie, G., Hoolst, T. V., Massé, M., Choblet, G., Lefèvre, A., Mitri, G., Baland, R.-M., Běhounková, M., Bourgeois, O., \& Trinh, A. (2016). Enceladus's internal ocean and ice shell constrained from Cassini gravity,

口 shape and libration data. Geophys. Res. Lett., 43, 5653-5660. doi 10.1002/ 2016GL068634.

Collins, G. C., \& Goodman, J. C. (2007). Enceladus' south polar sea. Icarus, 189, 72-82. doi:10.1016/j.icarus.2007.01.010.

Dermott, S. F., Malhotra, R., \& Murray, C. D. (1988). Dynamics of the Uranian and Saturnian satellite systems: A chaotic route to melting Miranda? Icarus, 76, 295-334. doi:10.1016/0019-1035(88)90074-7.

Durham, W. B., Stern, L. A., Kubo, T., \& Kirby, S. H. (2005). Flow strength of highly hydrated Mg- and Na-sulfate hydrate salts, pure and in mixtures 
with water ice, with application to Europa. J. Geophys. Res. Planets, 110. doi:10.1029/2005JE002475.

Fortes, A. D., \& Choukroun, M. (2010). Phase behaviour of ices and hydrates. Space Sci. Rev., 153, 185-218. doi 10.1007/s11214-010-9633-3.

380 Fuller, J., Luan, J., \& Quataert, E. (2016). Resonance locking as the source of rapid tidal migration in the Jupiter and Saturn moon systems. Mon. Not. R. Astron. Soc., . doi $10.1093 / \mathrm{mnras} / \mathrm{stw609}$

Gavrilov, S., \& Zharkov, V. (1977). Love numbers of the giant planets. Icarus, 32, 443-449. doi 10.1016/0019-1035(77)90015-X.

385 Goldsby, D. L., \& Kohlstedt, D. L. (2001). Superplastic deformation of ice: Experimental observations. J. Geophys. Res. Solid Earth, 106, 11017-11030. doi:10.1029/2000JB900336.

Howett, C. J. A., Spencer, J. R., Pearl, J., \& Segura, M. (2011). High heat flow from Enceladus' south polar region measured using 10-600

390 \ $\mathrm{cm}^{-1}$ Cassini/CIRS data. J. Geophys. Res. Planets, 116. doi 10.1029/ 2010JE003718,

Iess, L., Stevenson, D. J., Parisi, M., Hemingway, D., Jacobson, R. A., Lunine, J. I., Nimmo, F., Armstrong, J. W., Asmar, S. W., Ducci, M., \& Tortora, P. (2014). The gravity field and interior structure of Enceladus. Science, 344 , 78-80. doi $10.1126 /$ science.1250551.

Kamata, S., Matsuyama, I., \& Nimmo, F. (2015a). Tidal resonance in icy satellites with subsurface oceans. J. Geophys. Res. Planets, 120, 1528-1542. doi $10.1002 / 2015 \mathrm{JE} 004821$.

Kamata, S., \& Nimmo, F. (2014). Impact basin relaxation as a probe for the 400 thermal history of Pluto. J. Geophys. Res. Planets, 119, 2272-2289. doi 10. 1002/2014JE004679. 
Kamata, S., Sugita, S., \& Abe, Y. (2012). A new spectral calculation scheme for long-term deformation of Maxwellian planetary bodies. J. Geophys. Res. Planets, 117. doi 10.1029/2011JE003945.

Kamata, S., Sugita, S., Abe, Y., Ishihara, Y., Harada, Y., Morota, T., Namiki, N., Iwata, T., Hanada, H., Araki, H., Matsumoto, K., Tajika, E., Kuramoto, K., \& Nimmo, F. (2015b). The relative timing of Lunar Magma Ocean solidification and the Late Heavy Bombardment inferred from highly degraded

口 impact basin structures. Icarus, 250, 492-503. doi 10.1016/j.icarus. 2014. 12.025 ,

Lainey, V., Karatekin, Ö., Desmars, J., Charnoz, S., Arlot, J.-E., Emelyanov, N., Poncin-Lafitte, C. L., Mathis, S., Remus, F., Tobie, G., \& Zahn, J.-P. (2012). Strong tidal dissipation in Saturn and constraints on Enceladus' ther-

n mal state from astrometry. Astrophys. J., 752, 14. doi:10.1088/0004-637X/ $752 / 1 / 14$

Lewis, E. L., \& Perkin, R. G. (1986). Ice pumps and their rates. J. Geophys. Res. Oceans, 91, 11756-11762. doi 10.1029/JC091iC10p11756

Malamud, U., \& Prialnik, D. (2013). Modeling serpentinization: Applied to the

口 early evolution of Enceladus and Mimas. Icarus, 225, 763-774. doi 10.1016/ j.icarus.2013.04.024.

Marion, G., Kargel, J., Catling, D., \& Lunine, J. (2012). Modeling ammoniaammonium aqueous chemistries in the Solar System's icy bodies. Icarus, 220, 932-946. doi $10.1016 / \mathrm{j}$. icarus.2012.06.016

Matsuyama, I. (2014). Tidal dissipation in the oceans of icy satellites. Icarus, 425 242, 11-18. doi $10.1016 / \mathrm{j}$. icarus.2014.07.005.

McKenzie, D., Nimmo, F., Jackson, J. A., Gans, P. B., \& Miller, E. L. (2000). Characteristics and consequences of flow in the lower crust. J. Geophys. Res. Solid Earth, 105, 11029-11046. doi:10.1029/1999JB900446. 
McKinnon, W. B. (2015). Effect of Enceladus's rapid synchronous spin on

interpretation of Cassini gravity. Geophys. Res. Lett., 42, 2137-2143. doi 10. 1002/2015GL063384.

Meyer, J., \& Wisdom, J. (2007). Tidal heating in Enceladus. Icarus, 188, 535-539. doi:10.1016/j.icarus.2007.03.001

Nimmo, F., Barr, A. C., Běhounková, M., \& McKinnon, W. B. (submitted). Geophysics and tidal-thermal evolution of Enceladus. In P. Schenk, R. Binzel, R. Clark, C. Howett, A. Verbiscer, \& J. H. Waite (Eds.), Enceladus and the Icy Moons of Saturn. Tucson, USA: Univ. Arizona Press.

Nimmo, F., Bills, B. G., \& Thomas, P. C. (2011). Geophysical implications of the long-wavelength topography of the Saturnian satellites. J. Geophys. Res. Planets, 116. doi 10.1029/2011JE003835.

Ojakangas, G. W., \& Stevenson, D. J. (1989). Thermal state of an ice shell on Europa. Icarus, 81, 220-241. doi:10.1016/0019-1035(89)90052-3.

O'Neill, C., \& Nimmo, F. (2010). The role of episodic overturn in generating the surface geology and heat flow on Enceladus. Nature Geosci., 3, 88-91. doi: $10.1038 /$ ngeo731.

Porco, C. C., Helfenstein, P., Thomas, P. C., Ingersoll, A. P., Wisdom, J., West, R., Neukum, G., Denk, T., Wagner, R., Roatsch, T., Kieffer, S., Turtle, E., McEwen, A., Johnson, T. V., Rathbun, J., Veverka, J., Wilson, D., Perry, J., Spitale, J., Brahic, A., Burns, J. A., DelGenio, A. D., Dones, L., Murray, C. D., \& Squyres, S. (2006). Cassini observes the active south pole of Enceladus. Science, 311, 1393-1401. doi:10.1126/science.1123013.

Postberg, F., Kempf, S., Schmidt, J., Brilliantov, N., Beinsen, A., Abel, B., Buck, U., \& Srama, R. (2009). Sodium salts in E-ring ice grains from an ocean below the surface of Enceladus. Nature, 459, 1098-1101. doi10.1038/ nature08046. 
Roberts, J. H. (2015). The fluffy core of Enceladus. Icarus, 258, 54-66. doi 10. $1016 / j$. icarus.2015.05.033.

Roberts, J. H., \& Nimmo, F. (2008). Tidal heating and the long-term stability 1 of a subsurface ocean on Enceladus. Icarus, 194, 675-689. doi:10.1016/j.

Shoji, D., Hussmann, H., Sohl, F., \& Kurita, K. (2014). Non-steady state tidal

口 heating of Enceladus. Icarus, 235, 75-85. doi 10.1016/j.icarus.2014.03. 006.

Spencer, J. R., \& Nimmo, F. (2013). Enceladus: An active ice world in the

Saturn system. Ann. Rev. Earth Planet. Sci., 41, 693-717. doi 10.1146/ annurev-earth-050212-124025

Spencer, J. R., Pearl, J. C., Segura, M., Flasar, F. M., Mamoutkine, A., Romani, P., Buratti, B. J., Hendrix, A. R., Spilker, L. J., \& Lopes, R. M. C. (2006). Cassini encounters Enceladus: Background and the discovery of a south polar hot spot. Science, 311, 1401-1405.

Thomas, P., Tajeddine, R., Tiscareno, M., Burns, J., Joseph, J., Loredo, T., Helfenstein, P., \& Porco, C. (2016). Enceladus's measured physical libration requires a global subsurface ocean. Icarus, 264, 37-47. doi:10.1016/j. icarus.2015.08.037.

475 Travis, B., \& Schubert, G. (2015). Keeping Enceladus warm. Icarus, 250, 32-42. doi $10.1016 / \mathrm{j}$. icarus. 2014.11.017

Tyler, R. (2011). Tidal dynamical considerations constrain the state of an ocean on Enceladus. Icarus, 211, 770-779.

Tyler, R. H. (2008). Strong ocean tidal flow and heating on moons of the outer planets. Nature, 456, 770-772. doi doi:10.1038/nature07571.

Van Hoolst, T., Baland, R.-M., \& Trinh, A. (2016). The diurnal libration

口 and interior structure of Enceladus. Icarus, 27r, 311-318. doi 10.1016/j. icarus.2016.05.025. 
Waite, J. H., Lewis, W. S., Magee, B. A., Lunine, J. I., McKinnon, W. B., Glein, C. R., Mousis, O., Young, D. T., Brockwell, T., Westlake, J., Nguyen, M.-J., Teolis, B. D., Niemann, H. B., McNutt Jr, R. L., Perry, M., \& Ip, W.-H. (2009). Liquid water on Enceladus from observations of ammonia and ${ }^{40} \mathrm{Ar}$ in the plume. Nature, 460, 487-490. doi:10.1038/nature08153.

White, O. L., Schenk, P. M., \& Dombard, A. J. (2013). Impact basin relaxation 490 on Rhea and Iapetus and relation to past heat flow. Icarus, 223, 699-709. doi $10.1016 / j$. icarus.2013.01.013. 


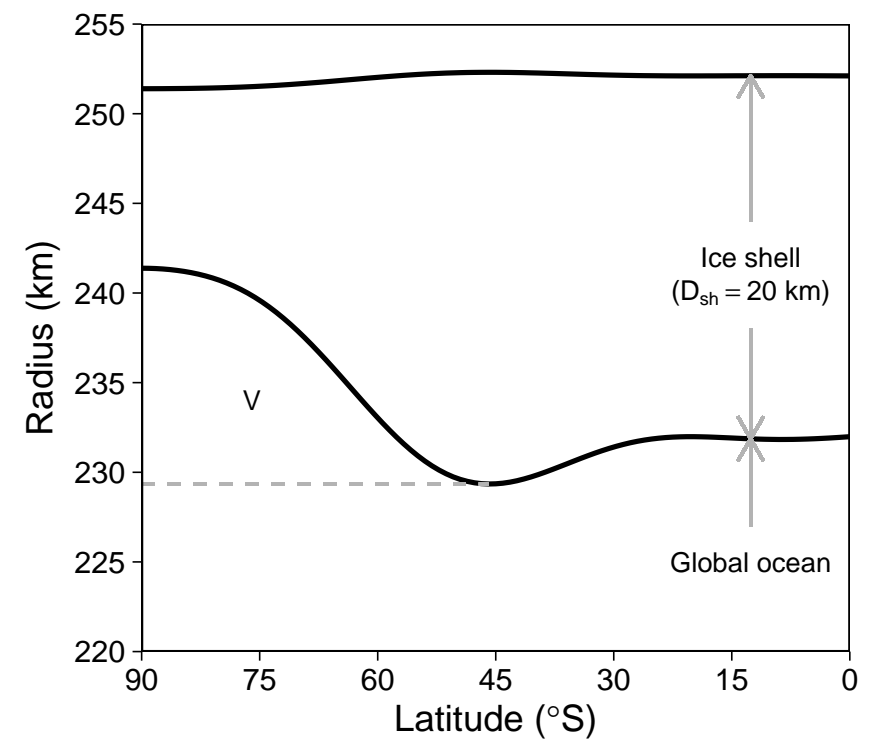

Figure 1: Cross section of the upper part of our Enceladus model. $V$ represents the volume of the thickened portion of the ocean.

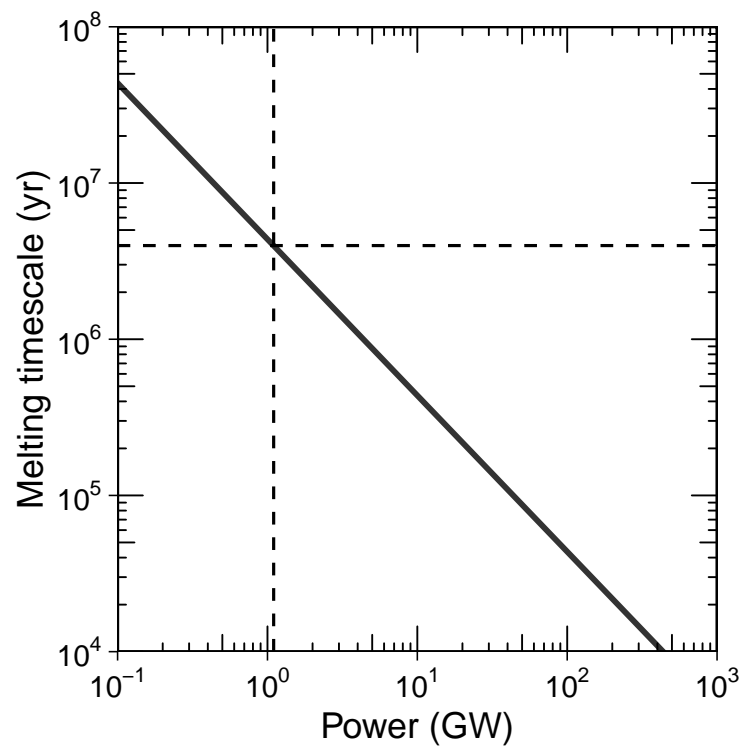

Figure 2: Timescale of melting as a function of power. A conventional estimate on the tidal heating rate (i.e., $1.1 \mathrm{GW}$ ) leads to a relaxation timescale of $\sim 4 \mathrm{Myr}$. 


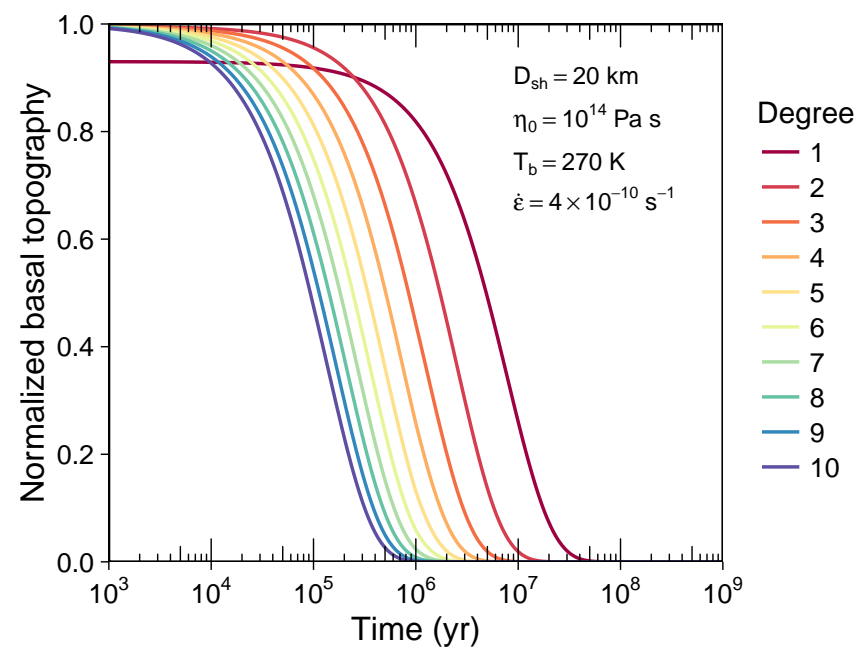

Figure 3: Time evolution of basal topography for different spherical harmonic degrees. Calculation conditions are shown. A higher degree results in a faster relaxation. Only degree-1 topography exhibits a non-negligible instant response because its boundary condition differs from those for other degrees.

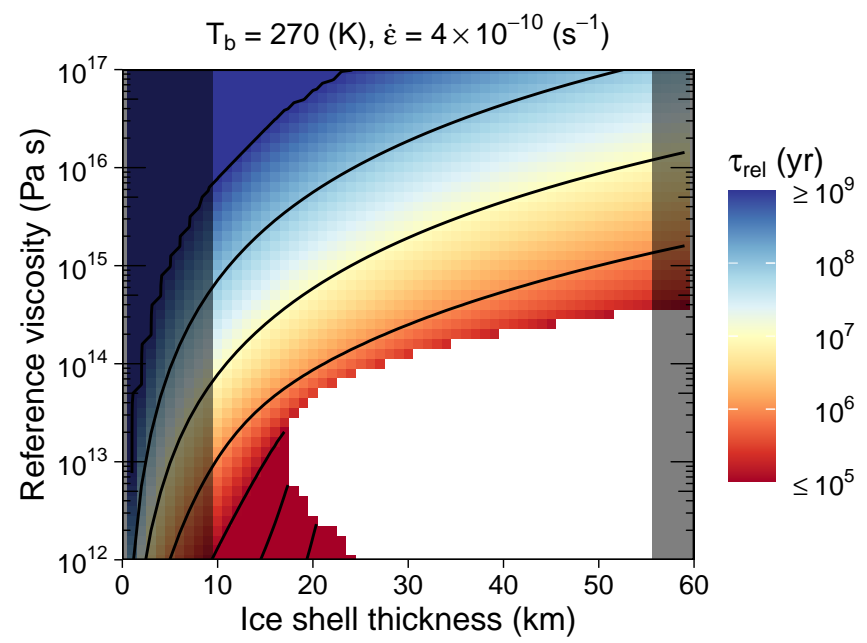

Figure 4: Relaxation timescale $\left(\tau_{\text {rel }}\right)$ as a function of ice shell thickness $\left(D_{\mathrm{sh}}\right)$ and reference viscosity of ice $\left(\eta_{\text {ref }}\right)$. Results for a basal temperature $\left(T_{b}\right)$ of $270 \mathrm{~K}$ and a strain rate $(\dot{\varepsilon})$ of $4 \times 10^{-10} \mathrm{~s}^{-1}$ are shown. The shaded areas in gray violate our assumptions that the ice shell is isostatically fully compensated. No solution for a large $D_{\mathrm{sh}}$ and a low $\eta_{\text {ref }}$ is found. 

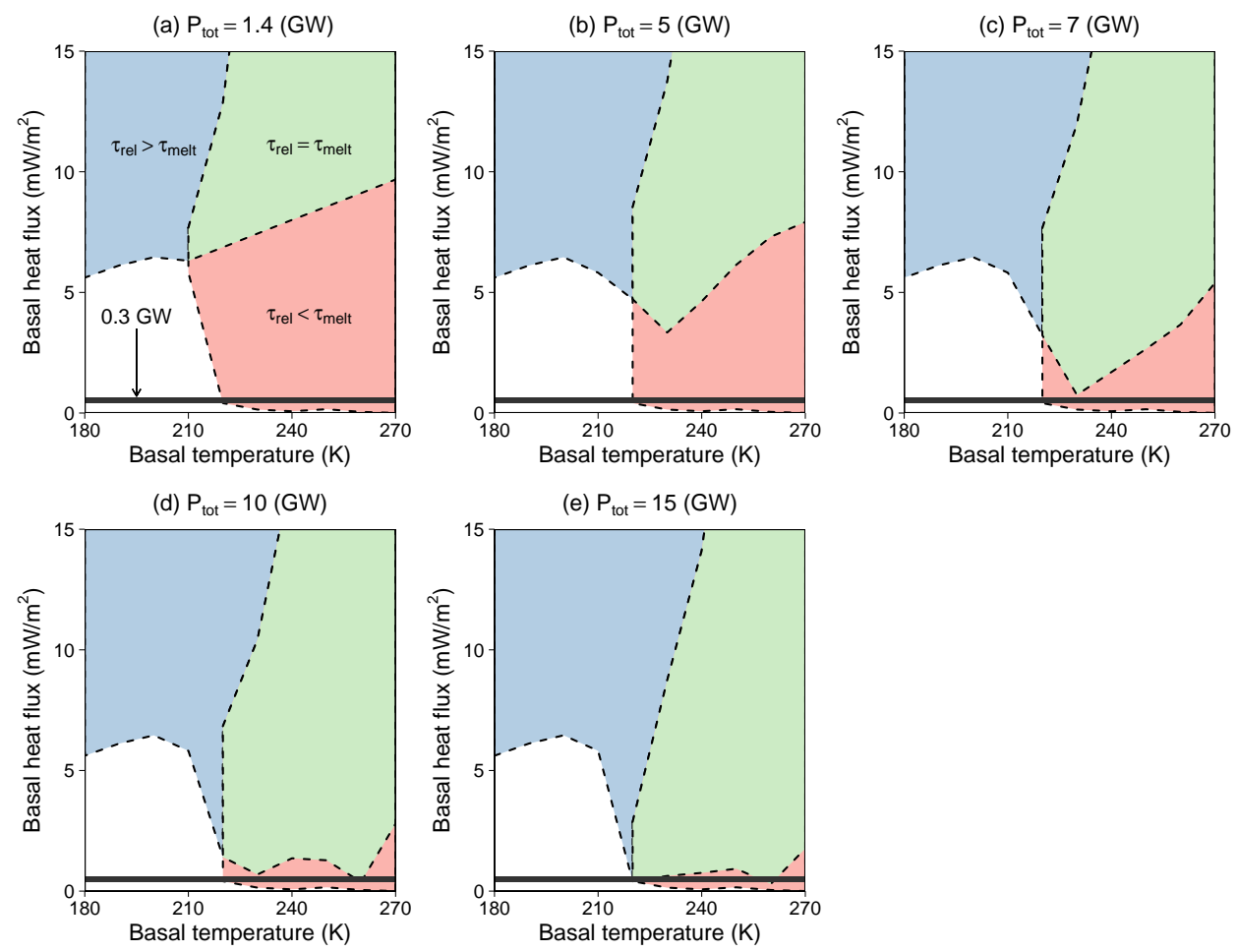

Figure 5: Comparison of the timescale of relaxation $\left(\tau_{\text {rel }}\right)$ and that of meting $\left(\tau_{\text {melt }}\right)$. Results under all calculation conditions are used to produce this figure. Each panel shows results for different powers $\left(P_{\text {tot }}=1.4-15 \mathrm{GW}\right)$. Parameter conditions that can lead to $\tau_{\text {rel }}=\tau_{\text {melt }}$ can be found only in the green area (upper right in each panel). The black band at $\sim 0.5 \mathrm{~mW} \mathrm{~m}^{-2}$ indicates the heat flux due to radiogenic heating in the core of $0.3 \mathrm{GW}$. No solution is found for a low basal temperature and a low basal heat flux. A basal heat flux due to radiogenic heating in the core can be sufficient for maintaining basal topography only if $P_{\text {tot }} \geq 10 \mathrm{GW}$. 


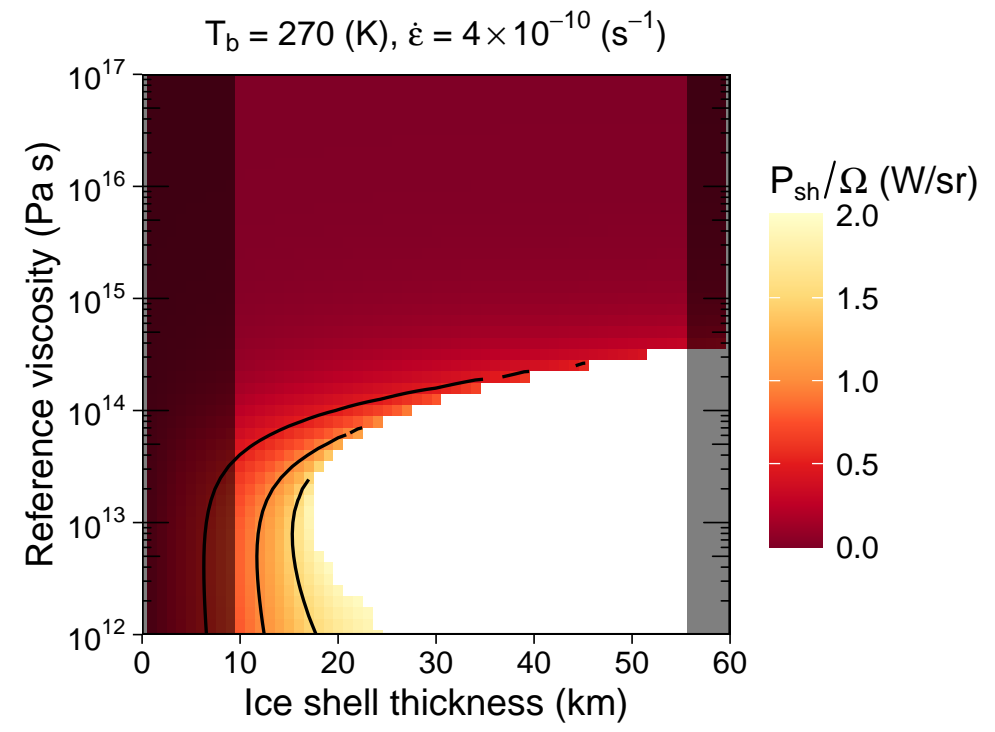

Figure 6: Power used for heating the ice shell $\left(P_{\mathrm{sh}}\right)$ as a function of ice shell thickness $\left(D_{\mathrm{sh}}\right)$ and reference viscosity of ice $\left(\eta_{\text {ref }}\right)$. Results for a basal temperature $\left(T_{b}\right)$ of $270 \mathrm{~K}$ and a strain rate $(\dot{\varepsilon})$ of $4 \times 10^{-10} \mathrm{~s}^{-1}$ are shown. The shaded areas in gray violate our assumptions that the ice shell is isostatically fully compensated. 
Table 1: Model parameters.

\begin{tabular}{llll}
\hline Symbol & Quantity & Value & Unit \\
\hline$R_{s}$ & Radius of Enceladus & 252.1 & $\mathrm{~km}$ \\
$D_{\mathrm{sh}}$ & Thickness of the ice shell & $10-56$ & $\mathrm{~km}$ \\
$g_{s}$ & Surface gravity & 0.11345 & $\mathrm{~m} \mathrm{~s}^{-2}$ \\
$\rho_{i}$ & Density of the ice shell & 920 & $\mathrm{~kg} \mathrm{~m}^{-3}$ \\
$\rho_{o}$ & Density of the ocean & 1000 & $\mathrm{~kg} \mathrm{~m}^{-3}$ \\
$\rho_{m}$ & Density of the mantle & 2470 & $\mathrm{~kg} \mathrm{~m}^{-3}$ \\
$\mu_{i}$ & Rigidity of the ice shell & 3.3 & $\mathrm{GPa}^{3}$ \\
$\mu_{m}$ & Rigidity of the mantle & 10 & $\mathrm{GPa}$ \\
$k$ & Thermal conductivity of ice & 2.3 & $\mathrm{~W} \mathrm{~m}{ }^{-1} \mathrm{~K}^{-1}$ \\
$L$ & Latent heat of ice & 330 & $\mathrm{~kJ} \mathrm{~kg}$ \\
$2 \pi / \omega$ & Orbital period & 1.37 & $\mathrm{days}$ \\
$T_{s}$ & Surface temperature & 75 & $\mathrm{~K}$ \\
$T_{\text {ref }}$ & Reference temperature & 273 & $\mathrm{~K}$ \\
$T_{b}$ & Basal temperature & $180-270$ & $\mathrm{~K}$ \\
$E_{a}$ & Activation energy & 60 & $\mathrm{~kJ} \mathrm{~mol}$ \\
$\eta_{\mathrm{ref}}$ & Reference viscosity of ice & $10^{12}-10^{17}$ & $\mathrm{~Pa} \mathrm{~s}$ \\
$\dot{\varepsilon}$ & Strain rate & $2 \times 10^{-10-5 \times 10^{-10}}$ & $\mathrm{~s}{ }^{-1}$ \\
\hline
\end{tabular}

